\title{
Recreational Travel Decisions: Push-Pull Dynamics on College Students
}

\author{
Ali Selman Özdemir ${ }^{1}$ \\ ${ }^{1}$ School of Physical Education and Sports, Cyprus International University, Nicosia, Cyprus \\ Correspondence: Ali Selman Özdemir, School of Physical Education and Sports, Cyprus International University, \\ Nicosia, Cyprus. E-mail: aozdemir@ciu.edu.tr
}

Received: December 12, 2019

Accepted: January 30, $2020 \quad$ Online Published: March 22, 2020

doi:10.5539/ies.v13n4p119

URL: https://doi.org/10.5539/ies.v13n4p119

\begin{abstract}
The purpose of this study is to examine the recreational tourism preferences of university students within the framework of their push and pull travel motivations. In addition, it was aimed to highlight how some variables and motivations affect tourism preferences in mutual interaction. Validity and reliability were obtained by "Scale of Recreational Activities in Destination Choices" (SRADC), "Scale of Intrinsic Travel Motivations" (SITM) and "Scale of Extrinsic Travel Motivations" (SETM) conducted by Özdemir, Karaküçük, and Büyüköztürk (2013). Descriptive statistics, independent sample $t$ test, one way ANOVA and Univariate test were used for data analysis, for in-group comparisons Tukey (HSD-LSD) and Pearson Correlation test were used. In this study, it was determined that .85 for recreational activities in destination choices scale, .90 for SITM and .91 for SETM. While the push and pull travel motivation of the participants was above the mean values $(123.96 \pm 15.65 ; 121.35 \pm$ 16.81), the highest subscale score in push travel motivation was obtained by the Exploring-Knowledge subdimension $(39.01 \pm 5.74)$. The highest sub-dimension score was obtained from the Escape sub-dimension $(38.31 \pm 5.98)$. In the pull travel motivation sub-dimensions, the highest sub-dimension score was the quality-atmosphere sub-dimension $(39.29 \pm 5.24)$, while the lowest sub-dimension score was obtained by the Natural Environment sub-dimension (19.98 \pm 4.58$)$. The findings of the study showed that the importance given to recreational activities was influenced by variables such as gender, year of study and perceived welfare, but also revealed differences in gender, field of study, year of study, and perceived welfare in push and pull travel motivations. In addition, there is a moderate positive relationship between recreational choices, push travel motivation and pull travel motivation. As a result, it has been determined that university students have high push and pull travel motivation and recreation preferences are differentiated between SITM and SETM by certain variables.
\end{abstract}

Keywords: recreational travel, push-pull, intrinsic-extrinsic, travel motivations, travel decision

\section{Introduction}

Recreation is defined as voluntary participation in leisure activities that are meaningful and enjoyable to the person involved (Cordes, 2013). Today the term recreation is used to describe activities or in a variety of structured settings as travel and tourism. The World Tourism Organization (1995) defined tourism as "travelling for leisure, business and other purposes for not more than one year". In tourism industry, travel is defined as "the act of moving outside one's community for business or pleasure but not for commuting or traveling to or from work or school" (Gee, Makens, \& Choy, 1989). Tourism basically concerns with people travelling from one place to another for pleasure or relaxation. Thereby, as an accepted knowledge, all tourism should have some travel, but not all travel is tourism.

To understand individuals travel decisions, especially the reasons for their chosen destinations and the recreational activities they participate in, motivations are the main factors that cause them to act. The dynamics of travel motivation has showed alternative ways to researchers to propound different views on travel motives. However, the main themes behind based on push and pull factors/motives.

The literature on tourist motivation indicates that the examination of motivations based on the push and pull factors have been generally accepted (Uysal, Li, \& Sirakaya-Turk, 2008). The notion of push and pull factors have been widely discussed and accepted as two core elements in estimating ones" travel motivation (Crompton, 1979; Dann, 1977). The push factors represent the needs and desires of the travelers and help to find answers to the reasons why 
people tend to escape where they live. Pull factors are the reasons that call us to the relevant destination. Push and pull factors have been generally characterized as relating two separate decisions made at two separate points in time. One focuses on whether to go and the other on where to go (Klenosky, 2002). Push factors refer to motivations such as escape from a perceived mundane environment, exploration and evaluation of self, relaxation, and regression, while pull factors relate to destinations, such as sunshine and friendly natives, to attract tourists and meet their needs (Dann, 1981). Thus, push factors motivate tourists to take a vacation and pull factors reinforce push factors. In other words, pull factors are the factors that attract travelers to choose a distinct destination. These factors combine the availability of beaches, cultural and historic heritage and even various attractions. In brief, these factors can be regarded as extrinsic motivations. Besides, push factors to involve forces an individual to take a trip to a particular destination. For instance, the need for rest, exploring and knowledge, escape, wellness, social communication, self-exploration or travel bragging, prestige.

Youth and student travel has become a global and fast-growing phenomenon (Richards \& Wilson, 2003). Particularly, university students represent an important segment for the tourism and leisure sectors (Kim, Oh, \& Jogaratnam, 2007). University student travelers are a subgroup of young tourists, as tourism is often an educational component of university experiences through spontaneous trips (e.g., independent trips), organized trips (e.g., field trips), or both. Independent trips among university students usually occur in a limited period in the context of a limited-term work or educational experience (Arnett, 2000).

Understanding this group's travel motivations - that is, their needs or desires that lead to specific travel behaviors including the style or way of trips they take, the destinations they choose to visit and the activities in which they prefer to attend (Biederman, 2008) when they are still students - is therefore critical. Moreover, researches on travel motivations and customer behaviors have focused on destination choice and paid little attention to motivation's importance and impact on activity preference. Studies on preferences for activities are important from a practical perspective. It informs tourism and recreation executives not only of their customers' choices, but also about the activities themselves and the physical conditions in which these activities take place (Jackson \& Schinkel, 1981).

According to activity-based model of decision making process for destination choice, motivation influences the preference of activities and the tourists will choose destinations depend on their preferred activities (Moscardo et al., 1996; Özdemir, Büyüköztürk, \& Karaküçük, 2016). This model not only clarifies the importance of activities in directing destination choice, but also states a direct relation between travel motivation and activity, for instance, among competition attendees (Kim, Sun, \& Mahoney, 2008).

Travel motivations has long been studied from different perspectives by using various approaches on several sample groups previously as (Crompton, 1979; Dann, 1977; Iso-Ahola, 1982; Pearce, 1993; Ryan \& Glendon, 1998) and recent studies as (Li, 2020; Phau et al., 2013; Rita, Brochado, \& Dimova, 2019; Yiamjanya \& Wongleedee, 2014; Xiao, So, \& Wang, 2015). In this study, our purpose to examine activity-based/recreational travel decisions in the light of push and pull factors (motivations) of university students in Turkey.

\section{Method}

\subsection{Study Sample}

The period in which the data were collected was from the end of March 2019 to the April 2019 at the province of Ankara and Zonguldak in Turkey. A simple random sampling method was used and 52 of the collected data were not evaluated in the research because of the missing. Aims of the study, the consent form, voluntary participation in the study, and confidentiality of the survey response were verbally explained by investigators before the distribution of the surveys to subjects. The survey were answered and completed within an average of 20 minutes. Finally, 498 university students from different faculties at Gazi University and Bülent Ecevit University participated in the study. It is observed that the majority of the participants are male (59.6\%), engineering students $(43.8 \%)$ and 4th-grade students (33.7\%).

\subsection{Instrumentation}

The questionnaire in this research consists of four parts. In the first section, there were seven closed-end questions for personal information of the students. The second, third, and fourth parts of the questionnaire included of the "Scale of Recreational Activities in Destination Choices" (SRADC) consisting 22 items, "Scale of Intrinsic Travel Motivations" (SITM) consisting 40 items, and "Scale of Extrinsic Travel Motivations" (SETM) consisting 40 items whose validity and reliability were confirmed by Özdemir, Karaküçük, and Büyüköztürk (2013). 4-point Likert scale was used in each answer option of the three scales. The ratings were determined as 1- Not Important at all; 2- Not important; 3-Important; and 4- Very Important, by 1 being the lowest and 4 being the highest scores. In 
this study, for each of the scales was satisfactory with Cronbach's alphas of .85 for recreational activities in destination choices scale, .90 for SITM and .91 for SETM.

\subsection{Data Analysis}

Data were analysed by using SPSS 24.0 version. The research has been prepared with the quantitative method, as a result of the analyses by skewness and kurtosis, it was determined that the data was homogeneously distributed. Descriptive statistics, independent sample $t$ test, one way ANOVA and Univariate test were used for data analysis, for in-group comparisons Tukey (HSD-LSD) and Pearson Correlation test were used.

\section{Results}

Table 1. Frequency and percentage range of demographic variables

\begin{tabular}{cccc}
\hline & $\mathrm{N}=(498)$ & & \\
\hline \multirow{3}{*}{ Gender } & Variable & $\mathrm{f}$ & $\%$ \\
\hline \multirow{3}{*}{ Area of education } & Female & 201 & 40.4 \\
& Male & 297 & 59.6 \\
& Social and Humanity Sciences & 185 & 37.1 \\
& Engineering & 218 & 43.8 \\
& Sports Sciences & 95 & 19.1 \\
\hline \multirow{3}{*}{ Year of education } & First year & 24 & 4.8 \\
& Second year & 96 & 19.3 \\
& Third year & 134 & 26.9 \\
& Fourth year & 168 & 33.7 \\
& Fifth year & 50 & 10.0 \\
& Sixth and above & 26 & 5.2 \\
\hline \multirow{3}{*}{ Perceived welfare } & Very poor & 21 & 4.2 \\
& Below Average & 62 & 12.4 \\
& Average & 282 & 56.6 \\
& Above Average & 107 & 21.5 \\
& Excellent & 26 & 5.2 \\
\hline
\end{tabular}

When the data of the demographic information of the participants are examined, it is observed that the majority of the participants are male (59.6\%), engineering (43.8\%), social and humanity science and sports science students $(19.1 \%)$ respectively, perceived an average welfare (56.6\%) and 4th-grade students $(33.7 \%)$.

Table 2. T-test results between participants' gender variable and recreational activities in destination choices

\begin{tabular}{cccccccc}
\hline & Gender & $\mathrm{N}$ & $\bar{x}$ & $\mathrm{ss}$ & $\mathrm{t}$ & $\mathrm{p}$ & Eta-Squ. \\
\hline \multirow{2}{*}{ Recreational Activities in Destination Choices } & Female & 201 & 2.86 & 0.43 & 2.259 & $0.02 *$ & 0.010 \\
& Male & 297 & 2.77 & 0.48 & & & \\
\hline
\end{tabular}

$* \mathrm{p}<0.05$.

According to the results, it is observed that female students give more importance to recreational activities than male students. Gender appears to have a low impact on recreational activities in destination choice.

Table 3. Anova test results between study area and recreational activities in destination choices

\begin{tabular}{cccccccc}
\hline & Study Area & $\mathrm{N}$ & $\bar{x}$ & ss & $\mathrm{F}$ & $\mathrm{p}$ & Eta-Squ. \\
\hline & Social and Humanity & 185 & $2.81 \mathrm{~b}$ & 0.42 & 51.059 & $0.000^{*}$ & 0.171 \\
& Engineering & 218 & $2.65 \mathrm{c}$ & 0.44 & & & \\
Recreational Activities in Destination Choices & Sports & 95 & $3.17 \mathrm{a}$ & 0.37 & & & \\
& Total & 498 & 2.81 & 0.46 & & & \\
& & & & &
\end{tabular}

$* \mathrm{p}<0.05 ; \mathrm{a}>\mathrm{b}>\mathrm{c}$.

There is a significant difference between the recreational activities in destination choices according to students' 
learning areas. When the difference between groups was analyzed, it was observed that sports science students had higher rates of recreational activities in destination choieces compared to other groups. However, when the level of importance was examined, no statistically significant difference was found.

Table 4. Anova test results between the year of study and recreational activities in destination choices

\begin{tabular}{cccccccc}
\hline & Year of Study & $\mathrm{N}$ & $\bar{x}$ & $\mathrm{ss}$ & $\mathrm{F}$ & $\mathrm{p}$ & Eta-Squ. \\
\hline & First year & 24 & 2.89 & 0.48 & 3.681 & $0.003^{*}$ & 0.036 \\
& Second year & 96 & 2.76 & 0.35 & & & \\
& Third year & 134 & 2.76 & 0.47 & & & \\
Recreational Activities in Destination Choices & Fourth year & 168 & 2.91 & 0.49 & & & \\
& Fifth year & 50 & 2.73 & 0.52 & & & \\
& Sixth and abo. & 26 & 2.61 & 0.29 & & & \\
& Total & 498 & 2.81 & 0.46 & & & \\
\hline
\end{tabular}

${ }^{*} \mathrm{p}<0.05 ; \mathrm{a}>\mathrm{b}>\mathrm{c}>\mathrm{d}>\mathrm{e}$.

It is seen that there is a significant difference between the importance level given to the recreational activities of the participants in the destination choices according to their education years. When the importance level that is below the average and the relations between the groups are analyzed, it is observed that the students whose on the fourth year of education give more importance to the recreational activities in the destination choice compared to other groups.

Table 5. Anova test results between perceived welfare and recreational activities in destination choices

\begin{tabular}{cccccccc}
\hline & Perceived Welfare & $\mathrm{N}$ & $\bar{x}$ & $\mathrm{ss}$ & $\mathrm{F}$ & $\mathrm{p}$ & Eta-Squ. \\
\hline & Very poor & 21 & 2.42 & 0.67 & 6.664 & $0.000^{*}$ & 0.051 \\
& Below Aver. & 62 & 2.72 & 0.43 & & & \\
Recreational Activities in Destination Choices & Average & 282 & 2.80 & 0.44 & & & \\
& Above Aver. & 107 & 2.93 & 0.42 & & & \\
& Excellent & 26 & 2.88 & 0.48 & & & \\
& Total & 498 & 2.81 & 0.46 & & & \\
\hline
\end{tabular}

$* \mathrm{p}<0.05 ; \mathrm{a}>\mathrm{b}>\mathrm{c}>\mathrm{d}>\mathrm{e}$.

When Table 5 is examined, a statistically significant difference was found between the level of perceived welfare by the participants and the recreational activities in the destination choices. As a result of the analysis of the differences in the group with post hoc tests, all the groups were found to be related to each other and it was seen that the participants who perceived the welfare as average showed an importance level compared to the other groups.

Table 6. Mean and standard deviation values of the push-pull motivations

\begin{tabular}{ccccc}
\hline \multicolumn{5}{c}{$\mathrm{N}=(498)$} \\
& Min. & Max. & $\chi$ & ss \\
\hline Total (Push Motives) & 78.00 & 160.00 & 123.96 & 15.65 \\
Exploring-Knowledge & 18.00 & 57.00 & 39.01 & 5.74 \\
Escape & 20.00 & 48.00 & 38.31 & 5.98 \\
Being Physically Active & 6.00 & 24.00 & 17.37 & 3.59 \\
Visiting Friends and Relatives & 5.00 & 20.00 & 14.49 & 3.05 \\
Travel Bragging-Prestige & 5.00 & 20.00 & 14.77 & 3.20 \\
Total (Pull Motives) & 64.00 & 160.00 & 121.35 & 16.81 \\
Quality-Atmosphere & 16.00 & 48.00 & 39.29 & 5.24 \\
Cultural\&Historic Heritage & 9.00 & 37.00 & 26.20 & 5.78 \\
Natural Environment & 7.00 & 28.00 & 19.98 & 4.58 \\
Popularity & 7.00 & 28.00 & 20.00 & 4.82 \\
\hline
\end{tabular}


While the push and pull travel motivation of the participants was above the average values $(123.96 \pm 15.65 ; 121.35$ \pm 16.81 ), the Exploring-Knowledge dimension received the highest sub-dimension score in push travel motivation $(39.01 \pm 5.74)$, and the second highest score obtained the Escape sub-dimension $(38.31 \pm 5.98)$. In the pull travel motivation sub-dimensions, the highest score was taken by the quality-atmosphere sub-dimension (39.29 \pm 5.24$)$, while the lowest sub-dimension score was obtained by the Authenticity sub-dimension (15.85 \pm 3.04$)$.

Table 7. T-test results between participants' gender variable and push-pull motivations

\begin{tabular}{|c|c|c|c|c|c|c|}
\hline & Gender & $\mathrm{N}$ & $\bar{x}$ & ss & $\mathrm{t}$ & $\mathrm{p}$ \\
\hline \multirow{2}{*}{ Total (Push Motives) } & Female & 201 & 124.18 & 14.15 & \multirow{2}{*}{0.256} & \multirow{2}{*}{0.798} \\
\hline & Male & 297 & 123.81 & 16.61 & & \\
\hline \multirow{2}{*}{ Exploring-Knowledge } & Female & 201 & 39.17 & 4.86 & \multirow{2}{*}{0.518} & \multirow{2}{*}{0.605} \\
\hline & Male & 297 & 38.90 & 6.26 & & \\
\hline \multirow{2}{*}{ Escape } & Female & 201 & 38.54 & 5.80 & \multirow{2}{*}{0.708} & \multirow{2}{*}{0.479} \\
\hline & Male & 297 & 38.15 & 6.10 & & \\
\hline \multirow{2}{*}{ Being Physically Active } & Female & 201 & 16.92 & 3.36 & \multirow{2}{*}{-2.231} & \multirow{2}{*}{$0.020^{*}$} \\
\hline & Male & 297 & 17.68 & 3.72 & & \\
\hline \multirow{2}{*}{ Visiting Friends and Relatives } & Female & 201 & 14.45 & 2.92 & \multirow{2}{*}{-0.248} & \multirow{2}{*}{0.804} \\
\hline & Male & 297 & 14.52 & 3.13 & & \\
\hline \multirow{2}{*}{ Travel Bragging-Prestige } & Female & 201 & 15.09 & 3.04 & \multirow{2}{*}{1.849} & \multirow{2}{*}{0.065} \\
\hline & Male & 297 & 14.55 & 3.28 & & \\
\hline \multirow{2}{*}{ Total (Pull Motives) } & Female & 201 & 122.75 & 14.23 & \multirow{2}{*}{1.532} & \multirow{2}{*}{0.126} \\
\hline & Male & 297 & 120.40 & 18.31 & & \\
\hline \multirow{2}{*}{ Quality-Atmosphere } & Female & 201 & 40.07 & 4.75 & \multirow{2}{*}{2.750} & \multirow{2}{*}{$0.006^{*}$} \\
\hline & Male & 297 & 38.77 & 5.49 & & \\
\hline \multirow{2}{*}{ Cultural\&Historic Heritage } & Female & 201 & 26.82 & 5.14 & \multirow{2}{*}{1.965} & \multirow{2}{*}{0.050} \\
\hline & Male & 297 & 25.79 & 6.14 & & \\
\hline \multirow{2}{*}{ Natural Environment } & Female & 201 & 20.00 & 4.25 & \multirow{2}{*}{0.056} & \multirow{2}{*}{0.955} \\
\hline & Male & 297 & 19.97 & 4.79 & & \\
\hline \multirow{2}{*}{ Popularity } & Female & 201 & 19.83 & 4.42 & \multirow{2}{*}{-0.658} & 0.11 \\
\hline & Male & 297 & 20.12 & 5.08 & & 0.011 \\
\hline A th otiot & Female & 201 & 16.01 & 2.74 & 0001 & 0.207 \\
\hline Authenticity & Male & 297 & 15.74 & 3.22 & 0.981 & 0.327 \\
\hline
\end{tabular}

${ }^{*} \mathrm{p}<0.05$.

As a result of the independent sample $t$ test conducted between the gender variable of the participants and push-pull motivations, a significant relation was found in the sub-dimension of being physically active in push travel motivation and in the atmosphere of quality atmosphere in the pull travel motivation. According to this, travel motivation levels were higher in favor of men in the being physically active sub-dimension and in favor of women in the quality atmosphere sub-dimension.

Table 8. Anova test results between study area and push-pull motivations

\begin{tabular}{|c|c|c|c|c|c|c|}
\hline & Study Area & $\mathrm{N}$ & $\bar{x}$ & ss & $\mathrm{F}$ & $\mathrm{p}$ \\
\hline \multirow{4}{*}{ Total (Push Motive) } & Social and Humanity Sciences & 185 & $123.23 \mathrm{~b}$ & 14.67 & \multirow{4}{*}{7.758} & \multirow{4}{*}{$0.000^{*}$} \\
\hline & Engineering & 218 & $122.17 \mathrm{c}$ & 15.96 & & \\
\hline & Sports Sciences & 95 & $129.49 \mathrm{a}$ & 15.69 & & \\
\hline & Total & 498 & 123.96 & 15.65 & & \\
\hline \multirow{4}{*}{ Exploring-Knowledge } & Social and Humanity Sciences & 185 & 39.13 & 5.66 & \multirow{4}{*}{0.612} & \multirow{4}{*}{0.543} \\
\hline & Engineering & 218 & 38.71 & 6.08 & & \\
\hline & Sports Sciences & 95 & 39.45 & 5.05 & & \\
\hline & Total & 498 & 39.01 & 5.74 & & \\
\hline
\end{tabular}




\begin{tabular}{|c|c|c|c|c|c|c|}
\hline \multirow{4}{*}{ Escape } & Social and Humanity Sciences & 185 & 38.00 & 5.84 & \multirow{4}{*}{0.631} & \multirow{4}{*}{0.533} \\
\hline & Engineering & 218 & 38.33 & 6.08 & & \\
\hline & Sports Sciences & 95 & 38.85 & 6.04 & & \\
\hline & Total & 498 & 38.31 & 5.98 & & \\
\hline \multirow{4}{*}{ Being Physically Active } & Social and Humanity Sciences & 185 & $17.08 \mathrm{~b}$ & 3.23 & \multirow{4}{*}{24.562} & \multirow{4}{*}{$0.000 *$} \\
\hline & Engineering & 218 & $16.66 \mathrm{c}$ & 3.76 & & \\
\hline & Sports Sciences & 95 & $19.56 \mathrm{a}$ & 3.02 & & \\
\hline & Total & 498 & 17.37 & 3.59 & & \\
\hline \multirow{4}{*}{ Visiting Friends and Relatives } & Social and Humanity Sciences & 185 & $14.07 \mathrm{c}$ & 2.92 & \multirow{4}{*}{7.904} & \multirow{4}{*}{$0.000^{*}$} \\
\hline & Engineering & 218 & $14.38 \mathrm{~b}$ & 2.94 & & \\
\hline & Sports Sciences & 95 & $15.55 \mathrm{a}$ & 3.28 & & \\
\hline & Total & 498 & 14.49 & 3.05 & & \\
\hline \multirow{4}{*}{ Travel Bragging-Prestige } & Social and Humanity Sciences & 185 & $14.94 \mathrm{~b}$ & 3.08 & \multirow{4}{*}{14.038} & \multirow{4}{*}{$0.000 *$} \\
\hline & Engineering & 218 & $14.06 \mathrm{c}$ & 3.38 & & \\
\hline & Sports Sciences & 95 & $16.06 \mathrm{a}$ & 2.46 & & \\
\hline & Total & 498 & 14.77 & 3.20 & & \\
\hline \multirow{4}{*}{$\begin{array}{c}\text { Total } \\
\text { (Pull Motives) }\end{array}$} & Social and Humanity Sciences & 185 & $122.76 \mathrm{~b}$ & 15.48 & \multirow{4}{*}{13.080} & \multirow{4}{*}{$0.000 *$} \\
\hline & Engineering & 218 & $117.52 \mathrm{c}$ & 17.78 & & \\
\hline & Sports Sciences & 95 & $127.41 \mathrm{a}$ & 14.84 & & \\
\hline & Total & 498 & 121.35 & 16.81 & & \\
\hline \multirow{4}{*}{ Quality-Atmosphere } & Social and Humanity Sciences & 185 & 39.83 & 5.17 & \multirow{4}{*}{1.558} & \multirow{4}{*}{0.212} \\
\hline & Engineering & 218 & 38.96 & 5.64 & & \\
\hline & Sports Sciences & 95 & 39.01 & 4.29 & & \\
\hline & Total & 498 & 39.29 & 5.24 & & \\
\hline \multirow{4}{*}{ Cultural \& Historic Heritage } & Social and Humanity Sciences & 185 & $26.67 \mathrm{~b}$ & 5.63 & \multirow{4}{*}{9.938} & \multirow{4}{*}{$0.000 *$} \\
\hline & Engineering & 218 & $25.03 \mathrm{c}$ & 6.09 & & \\
\hline & Sports Sciences & 95 & $27.98 \mathrm{a}$ & 4.70 & & \\
\hline & Total & 498 & 26.20 & 5.78 & & \\
\hline \multirow{4}{*}{ Natural Environment } & Social and Humanity Sciences & 185 & $19.81 \mathrm{~b}$ & 4.39 & \multirow{4}{*}{7.002} & \multirow{4}{*}{$0.001 *$} \\
\hline & Engineering & 218 & $19.46 \mathrm{c}$ & 4.95 & & \\
\hline & Sports Sciences & 95 & $21.51 \mathrm{a}$ & 3.67 & & \\
\hline & Total & 498 & 19.98 & 4.58 & & \\
\hline \multirow{4}{*}{ Popularity } & Social and Humanity Sciences & 185 & $20.37 b$ & 4.51 & \multirow{4}{*}{21.565} & \\
\hline & Engineering & 218 & $18.67 \mathrm{c}$ & 5.13 & & $0000 *$ \\
\hline & Sports Sciences & 95 & $22.33 \mathrm{a}$ & 3.52 & & \\
\hline & Total & 498 & 20.00 & 4.82 & & \\
\hline & Social and Humanity Sciences & 185 & $16.05 \mathrm{~b}$ & 2.87 & & \\
\hline A thanticity & Engineering & 218 & $15.38 \mathrm{c}$ & 3.36 & 5.715 & $0004 *$ \\
\hline Autnenticity & Sports Sciences & 95 & $16.55 \mathrm{a}$ & 2.31 & 5.115 & $0.0044^{4}$ \\
\hline & Total & 498 & 15.85 & 3.04 & & \\
\hline
\end{tabular}

$* \mathrm{p}<0.05$.

According to the results of the Anova test conducted between the push and pull travel motivations of the participants and their study areas; There is a statistically significant difference in all sub-dimensions and total scores except for Exploring-Knowledge, escape, and quality-atmosphere subscales. In all groups with significant differences, it was found that sports science students achieved higher scores in their push travel motivations and pull travel motivations than students in other fields of study. Except for the sports science $>$ engineering $>$ social and humanities rankings in the Visiting Friends and Relatives sub-dimension, which is one of the push travel motivation sub-dimensions in the group comparisons, the ranking in all other group comparisons is sports science> social and humanities $>$ engineering. 
Table 9. Anova test results between year of education and push-pull motivations

\begin{tabular}{|c|c|c|c|c|c|c|}
\hline & Year of Education & $\mathrm{N}$ & $\bar{x}$ & ss & $\mathrm{F}$ & $\mathrm{p}$ \\
\hline \multirow{7}{*}{ Total (Push Motive) } & First year & 24 & 127.41 & 14.36 & \multirow{7}{*}{1.758} & \multirow{7}{*}{0.120} \\
\hline & Second year & 96 & 124.76 & 13.18 & & \\
\hline & Third year & 134 & 121.51 & 15.31 & & \\
\hline & Fourth year & 168 & 125.92 & 16.25 & & \\
\hline & Fifth year & 50 & 121.80 & 16.61 & & \\
\hline & Sixth and above & 26 & 121.96 & 19.57 & & \\
\hline & Total & 498 & 123.96 & 15.65 & & \\
\hline \multirow{7}{*}{ Exploring-Knowledge } & First year & 24 & 40.70 & 5.44 & \multirow{7}{*}{1.022} & \multirow{7}{*}{0.404} \\
\hline & Second year & 96 & 39.00 & 5.28 & & \\
\hline & Third year & 134 & 38.56 & 6.17 & & \\
\hline & Fourth year & 168 & 39.39 & 5.74 & & \\
\hline & Fifth year & 50 & 38.80 & 5.28 & & \\
\hline & Sixth and above & 26 & 37.69 & 6.06 & & \\
\hline & Total & 498 & 39.01 & 5.74 & & \\
\hline \multirow{7}{*}{ Escape } & First year & 24 & 37.66 & 6.23 & \multirow{7}{*}{.838} & \multirow{7}{*}{0.523} \\
\hline & Second year & 96 & 38.73 & 5.00 & & \\
\hline & Third year & 134 & 37.68 & 5.62 & & \\
\hline & Fourth year & 168 & 38.79 & 6.54 & & \\
\hline & Fifth year & 50 & 37.60 & 6.68 & & \\
\hline & Sixth and above & 26 & 38.76 & 5.78 & & \\
\hline & Total & 498 & 38.31 & 5.98 & & \\
\hline \multirow{7}{*}{ Being Physically Active } & First year & 24 & 18.37 & 2.53 & \multirow{7}{*}{1.868} & \multirow{7}{*}{0.098} \\
\hline & Second year & 96 & 17.28 & 3.16 & & \\
\hline & Third year & 134 & 16.91 & 3.31 & & \\
\hline & Fourth year & 168 & 17.83 & 3.83 & & \\
\hline & Fifth year & 50 & 17.34 & 3.58 & & \\
\hline & Sixth and above & 26 & 16.26 & 5.18 & & \\
\hline & Total & 498 & 17.37 & 3.59 & & \\
\hline \multirow{7}{*}{ Visiting Friends and Relatives } & First year & 24 & 15.62 & 3.32 & \multirow{7}{*}{1.730} & \multirow{7}{*}{0.126} \\
\hline & Second year & 96 & 14.53 & 2.95 & & \\
\hline & Third year & 134 & 14.17 & 3.02 & & \\
\hline & Fourth year & 168 & 14.79 & 3.10 & & \\
\hline & Fifth year & 50 & 13.96 & 2.50 & & \\
\hline & Sixth and above & 26 & 14.03 & 3.63 & & \\
\hline & Total & 498 & 14.49 & 3.05 & & \\
\hline \multirow{7}{*}{ Travel Bragging-Prestige } & First year & 24 & 15.04 & 3.71 & \multirow{7}{*}{2.249} & \multirow{7}{*}{$.048^{*}$} \\
\hline & Second year & 96 & $15.20 \mathrm{a}$ & 3.17 & & \\
\hline & Third year & 134 & $14.17 \mathrm{c}$ & 3.19 & & \\
\hline & Fourth year & 168 & $15.10 \mathrm{~b}$ & 2.96 & & \\
\hline & Fifth year & 50 & $14.10 \mathrm{~d}$ & 3.44 & & \\
\hline & Sixth and above & 26 & 15.19 & 3.40 & & \\
\hline & Total & 498 & 14.77 & 3.20 & & \\
\hline \multirow{7}{*}{ Total (Pull Motives) } & First year & 24 & 121.45 & 17.08 & & \\
\hline & Second year & 96 & $120.08 \mathrm{~b}$ & 17.09 & & \\
\hline & Third year & 134 & $118.83 \mathrm{c}$ & 16.13 & & \\
\hline & Fourth year & 168 & $124.84 \mathrm{a}$ & 17.07 & 2.581 & $0.026^{*}$ \\
\hline & Fifth year & 50 & $118.18 \mathrm{~d}$ & 15.85 & & \\
\hline & Sixth and above & 26 & 122.50 & 16.45 & & \\
\hline & Total & 498 & 121.35 & 16.81 & & \\
\hline & First year & 24 & 39.20 & 3.99 & & \\
\hline Quality-Atmosphere & Second year & 96 & 39.12 & 5.20 & 1.409 & 0.219 \\
\hline & Third year & 134 & 38.48 & 5.44 & & \\
\hline
\end{tabular}




\begin{tabular}{|c|c|c|c|c|c|c|}
\hline & Fourth year & 168 & 40.06 & 5.06 & & \\
\hline & Fifth year & 50 & 39.16 & 5.70 & & \\
\hline & Sixth and above & 26 & 39.53 & 5.39 & & \\
\hline & Total & 498 & 39.29 & 5.24 & & \\
\hline \multirow{7}{*}{ Cultural\&Historic Heritage } & First year & 24 & 26.16 & 6.83 & \multirow{7}{*}{1.911} & \multirow{7}{*}{0.091} \\
\hline & Second year & 96 & 26.19 & 5.88 & & \\
\hline & Third year & 134 & 25.46 & 5.79 & & \\
\hline & Fourth year & 168 & 27.03 & 5.81 & & \\
\hline & Fifth year & 50 & 24.86 & 5.15 & & \\
\hline & Sixth and above & 26 & 27.38 & 4.54 & & \\
\hline & Total & 498 & 26.20 & 5.78 & & \\
\hline \multirow{7}{*}{ Natural Environment } & First year & 24 & 21.00 & 3.83 & \multirow{7}{*}{3.124} & \multirow{7}{*}{$0.009 *$} \\
\hline & Second year & 96 & $19.31 \mathrm{~d}$ & 4.72 & & \\
\hline & Third year & 134 & $19.58 \mathrm{c}$ & 4.40 & & \\
\hline & Fourth year & 168 & $20.70 \mathrm{~b}$ & 4.61 & & \\
\hline & Fifth year & 50 & $18.68 \mathrm{e}$ & 4.84 & & \\
\hline & Sixth and above & 26 & $21.50 \mathrm{a}$ & 3.89 & & \\
\hline & Total & 498 & 19.98 & 4.58 & & \\
\hline \multirow{7}{*}{ Popularity } & First year & 24 & 19.29 & 6.18 & \multirow{7}{*}{2.384} & \multirow{7}{*}{$0.037 *$} \\
\hline & Second year & 96 & $19.48 \mathrm{c}$ & 4.96 & & \\
\hline & Third year & 134 & $19.60 \mathrm{~b}$ & 4.58 & & \\
\hline & Fourth year & 168 & $20.92 a$ & 4.46 & & \\
\hline & Fifth year & 50 & 20.18 & 4.67 & & \\
\hline & Sixth and above & 26 & $18.34 \mathrm{~d}$ & 5.91 & & \\
\hline & Total & 498 & 20.00 & 4.82 & & \\
\hline \multirow{7}{*}{ Authenticity } & First year & 24 & 15.79 & 2.91 & \multirow{7}{*}{.686} & \multirow{7}{*}{.635} \\
\hline & Second year & 96 & 15.95 & 3.16 & & \\
\hline & Third year & 134 & 15.70 & 3.10 & & \\
\hline & Fourth year & 168 & 16.11 & 2.87 & & \\
\hline & Fifth year & 50 & 15.30 & 3.32 & & \\
\hline & Sixth and above & 26 & 15.73 & 2.89 & & \\
\hline & Total & 498 & 15.85 & 3.04 & & \\
\hline
\end{tabular}

$* \mathrm{p}<0.05$

As a result of the anova test comparing the education year of the participants with the push and pull travel motivations, statistically significant differences were found for the Travel Bragging-Prestige in push travel motivations, in the external travel motivations, in the total scores and Natural Environment and popularity sub-dimensions. When we examine the comparisons within the group; Intra-group relationships realized as $2>3>$ $4>5$ were determined as $4>2>3>5$ in total pull travel motivation total scores. Intra-group relationships determined as $6>4>3>2>5$ in the Natural Environment sub-dimension were determined as $4>3>2>6$.

Table 10. Anova test results between percieved welfare and push-pull motivations

\begin{tabular}{|c|c|c|c|c|c|c|}
\hline & Percieved Welfare & $\mathrm{N}$ & $\bar{x}$ & ss & $\mathrm{F}$ & $\mathrm{p}$ \\
\hline \multirow{6}{*}{ Total (Push Motive) } & Very poor & 21 & 119.85 & 16.84 & \multirow{6}{*}{1.001} & \multirow{6}{*}{0.406} \\
\hline & Below Average & 62 & 122.45 & 13.78 & & \\
\hline & Average & 282 & 123.85 & 15.60 & & \\
\hline & Above Average & 107 & 125.02 & 15.42 & & \\
\hline & Excellent & 26 & 127.69 & 19.93 & & \\
\hline & Total & 498 & 123.96 & 15.65 & & \\
\hline \multirow{4}{*}{ Exploring-Knowledge } & Very poor & 21 & 37.04 & 7.24 & \multirow{4}{*}{0.751} & \multirow{4}{*}{0.558} \\
\hline & Below Average & 62 & 39.30 & 4.48 & & \\
\hline & Average & 282 & 39.16 & 5.82 & & \\
\hline & Above Average & 107 & 38.78 & 5.45 & & \\
\hline
\end{tabular}




\begin{tabular}{|c|c|c|c|c|c|c|}
\hline & Excellent & 26 & 39.19 & 7.27 & & \\
\hline & Total & 498 & 39.01 & 5.74 & & \\
\hline \multirow{6}{*}{ Escape } & Very poor & 21 & 38.09 & 7.58 & \multirow{6}{*}{0.132} & \multirow{6}{*}{0.971} \\
\hline & Below Average & 62 & 38.77 & 6.81 & & \\
\hline & Average & 282 & 38.20 & 5.50 & & \\
\hline & Above Average & 107 & 38.28 & 6.22 & & \\
\hline & Excellent & 26 & 38.57 & 6.88 & & \\
\hline & Total & 498 & 38.31 & 5.98 & & \\
\hline \multirow{6}{*}{ Being Physically Active } & Very poor & 21 & $16.38 \mathrm{e}$ & 3.58 & \multirow{6}{*}{6.358} & \multirow{6}{*}{$0.000^{*}$} \\
\hline & Below Average & 62 & $15.77 \mathrm{~d}$ & 3.20 & & \\
\hline & Average & 282 & $17.32 \mathrm{c}$ & 3.77 & & \\
\hline & Above Average & 107 & $18.30 \mathrm{~b}$ & 3.03 & & \\
\hline & Excellent & 26 & $18.65 \mathrm{a}$ & 3.19 & & \\
\hline & Total & 498 & 17.37 & 3.59 & & \\
\hline \multirow{6}{*}{ Visiting Friends and Relatives } & Very poor & 21 & 14.61 & 3.05 & \multirow{6}{*}{2.318} & \multirow{6}{*}{0.056} \\
\hline & Below Average & 62 & 13.87 & 3.16 & & \\
\hline & Average & 282 & 14.59 & 3.06 & & \\
\hline & Above Average & 107 & 14.22 & 2.74 & & \\
\hline & Excellent & 26 & 15.88 & 3.52 & & \\
\hline & Total & 498 & 14.49 & 3.05 & & \\
\hline \multirow{6}{*}{ Travel Bragging-Prestige } & Very poor & 21 & 13.71 & 2.14 & \multirow{6}{*}{2.245} & \multirow{6}{*}{0.063} \\
\hline & Below Average & 62 & 14.72 & 3.40 & & \\
\hline & Average & 282 & 14.56 & 3.12 & & \\
\hline & Above Average & 107 & 15.42 & 3.13 & & \\
\hline & Excellent & 26 & 15.38 & 4.06 & & \\
\hline & Total & 498 & 14.77 & 3.20 & & \\
\hline \multirow{6}{*}{ Total (Pull Motives) } & Very poor & 21 & 114.00 & 22.95 & \multirow{6}{*}{1.147} & \multirow{6}{*}{0.334} \\
\hline & Below Average & 62 & 120.80 & 17.49 & & \\
\hline & Average & 282 & 121.56 & 16.73 & & \\
\hline & Above Average & 107 & 122.40 & 15.67 & & \\
\hline & Excellent & 26 & 122.00 & 14.42 & & \\
\hline & Total & 498 & 121.35 & 16.81 & & \\
\hline \multirow{6}{*}{ Quality-Atmosphere } & Very poor & 21 & 37.00 & 8.42 & \multirow{6}{*}{1.635} & \multirow{6}{*}{0.164} \\
\hline & Below Average & 62 & 38.91 & 5.19 & & \\
\hline & Average & 282 & 39.70 & 5.32 & & \\
\hline & Above Average & 107 & 38.97 & 4.47 & & \\
\hline & Excellent & 26 & 39.03 & 3.70 & & \\
\hline & Total & 498 & 39.29 & 5.24 & & \\
\hline \multirow{6}{*}{ Cultural \& Historic Heritage } & Very poor & 21 & 24.38 & 5.92 & & \\
\hline & Below Average & 62 & 25.79 & 5.70 & & \\
\hline & Average & 282 & 26.52 & 5.71 & 0876 & 0.478 \\
\hline & Above Average & 107 & 25.94 & 5.96 & 0.070 & \\
\hline & Excellent & 26 & 26.34 & 5.77 & & \\
\hline & Total & 498 & 26.20 & 5.78 & & \\
\hline & Very poor & 21 & 19.80 & 5.44 & & \\
\hline & Below Average & 62 & 20.09 & 4.96 & & \\
\hline Natural Environment & Average & 282 & 19.86 & 4.67 & 0.214 & 0.930 \\
\hline & Above Average & 107 & 20.31 & 4.10 & & \\
\hline & Excellent & 26 & 19.80 & 3.98 & & \\
\hline & Total & 498 & 19.98 & 4.58 & & \\
\hline & Very poor & 21 & 18.76 & 5.70 & & \\
\hline Ponularity & Below Average & 62 & 19.61 & 5.49 & 2357 & 0053 \\
\hline D & Average & 282 & 19.68 & 4.81 & & \\
\hline & Above Average & 107 & 21.09 & 4.46 & & \\
\hline
\end{tabular}




\begin{tabular}{|c|c|c|c|c|c|c|}
\hline & Excellent & 26 & 20.88 & 3.10 & & \\
\hline & Total & 498 & 20.00 & 4.82 & & \\
\hline \multirow{6}{*}{ Authenticity } & Very poor & 21 & $14.04 \mathrm{e}$ & 3.66 & \multirow{6}{*}{2.539} & \multirow{6}{*}{$0.039 *$} \\
\hline & Below Average & 62 & $16.38 \mathrm{a}$ & 2.87 & & \\
\hline & Average & 282 & $15.78 \mathrm{~d}$ & 3.13 & & \\
\hline & Above Average & 107 & $16.07 \mathrm{~b}$ & 2.78 & & \\
\hline & Excellent & 26 & $15.92 \mathrm{c}$ & 2.41 & & \\
\hline & Total & 498 & 15.85 & 3.04 & & \\
\hline
\end{tabular}

$* \mathrm{p}<0.05$.

As a result of the analysis between the perceived welfare variable and the push-pull travel motivations, there was a significant difference in the push travel motivation in the being physically active sub-dimension and in the pull travel motivation sub-dimension in the Authenticity sub-dimension. In the sub-dimension of the being physically active, it is seen that all perceived welfare options are related to each other, whereas in this relationship, the group which perceived welfare level Excellent achieved the highest average, the being physically active points decrease in parallel with the perceived welfare level. Similarly, in the sub-dimension of Authenticity, , it is seen that all perceived welfare options are related to each other, whereas in this relationship, the group which perceived welfare level Very Poor achieved the highest average.

Table 11. Pearson correlation test results between recreational activities in destination choices and push-pull travel motivation

\begin{tabular}{ccccc}
\hline & & Recreation Activities & Push Motives & Pull Motives \\
\hline \multirow{4}{*}{ Recreational Activities } & Pearson Correlation & 1 & & \\
& Sig. (2-tailed) & & & \\
& $\mathrm{N}$ & 498 & & \\
\hline \multirow{2}{*}{ Push Motivations } & Pearson Correlation &, $602^{* *}$ & 1 & \\
& Sig. (2-tailed) &, 000 & & \\
& $\mathrm{~N}$ & 498 & 498 & \multirow{2}{*}{, $065^{* *}$} \\
\hline \multirow{2}{*}{ Pull Motivations } & Pearson Correlation &, $549^{* *}$ &, 000 & \\
$\mathrm{p}>0.01 ; 0.05$ & Sig. (2-tailed) &, 000 & 498 & 498 \\
\hline
\end{tabular}

When the relationship between the level of importance given to recreational activities in the destination choices and the push-pull travel motivations; It has been determined that the importance given to recreational activities has a positive and moderate relationship with both push-pull travel motivations. Therefore, based on the findings obtained, as the motivation of push-pull travel motivations increases, the importance given to recreational activities also increases. Likewise, as the level of importance given to recreational activities increases, it can be said that push-pull travel motivations will be positively affected.

\section{Discussion and Conclusion}

The purpose of this study is to examine the recreational travel decisions of university students within the perspective of push and pull travel motivations. In addition, it was aimed to highlight the relationship between motivations, recreational activities and various personal variables. While the push and pull travel motivation of the participants was above the average values; the Exploring-Knowledge and Escape sub-dimensions received the highest scores in push travel motivation. Visiting Friends and Relatives and Travel Bragging-Prestige sub-dimensions were similarly had lowest scores. In the sub-dimensions of pull travel motivations, the highest scores were taken by the Quality-Atmosphere and Cultural \& Historic Heritage while the lowest score was obtained by the Authenticity. Richards and Wilson (2003) gathered international information on the independent travel market for the youth and student. Responses were taken from 2300 youth including students from Canada, Hong Kong, Mexico, Slovenia, South Africa, Sweden, the Czech Republic and the UK. While study participants appeared to have lower incomes, they were obviously prepared to save and/or work during their travels in order to increase their spending power considerably. Their main motivation was to discover other cultures, followed by curiosity and increased knowledge. In the study of Kim et al. (2007), knowledge, sports and adventure are indicated as significant motivational push factors for US students. In another study, some of the most significant 
push motivations for Omani students are 'to be mentally refreshed,' 'to learn something new or to increase knowledge' and 'to relax,' as stated by Mohsin and Alsawafi (2011). Escapism is a major motivation found in study by Thrane (2008). For both UK and Chinese students, 'to relax' and 'discovering something new' are considered important motivations in travel (Xu et al., 2009). However, a study conducted by Asian international and American domestic students found that knowledge, sports, entertainment, relaxation, leisure, family and travel bragging were major motives for travel, but differed by culture (Kim \& Jogaratnam, 2002). Asian international students, for instance, rated knowledge as more relevant, while American domestic students rated sports, entertainment, relaxation, family and travel bragging as more significant. While focused on millennials' cross-cultural differences; Rita, Brochado, and Dimova (2019) concluded that the most important motivations for travelling are 'to relax', followed closely by the desire 'to escape from the ordinary', while the least important motivations are travelling 'to meet people with similar interests' and 'to go to places my friends have not previously visited'. Accordind to Kim and Lee (2000), American travelers exhibit more individualistic features whereas their Japanese counterparts exhibit more collectivistic features. Also, the two groups of travelers varied in their motivations as prestige/status, family togetherness, and novelty. Likewise, differences in culture and values contribute to a range of preferences for travel amongst university students. Japanese student travelers are more collectivistic, psychocentric than American students and likely to visit popular destinations (Sakakida et al., 2004). A comparative study of US, South African and Israeli students reported differences in transportation, food, leisure activity, and accommodation choices (Shoham et al., 2005). Additionally, several studies showed variations in student travel behaviors such as destination choices, use of travel services and motives (Field, 1999; Wang \& Walker, 2010; Xu et al., 2009).

According to the results, it is observed that female students give more importance to recreational activities than male students. Gender appears to have a low impact on recreational activities in destination choice. Nonetheless, the studies of Kim and Jogaratnam (2002) and Xu et al. (2009) showed that males tend to be more active than females do, and that males often engage more often in outdoor and sport activities. Carr (1999) studied the behavior of young beach-oriented tourists in the UK, and apparently detected little variation by gender in young tourists' leisure activities. The only significant difference was noticed with shopping, which is more appealing to young women visitors. As a result of the gender variable and push-pull motivations differences, a significant relation was found in the sub-dimension of being physically active in push travel motivation and in the atmosphere of quality atmosphere in the pull travel motivation. According to this, travel motivation levels were higher in favor of men in the being physically active sub-dimension and in favor of women in the quality atmosphere sub-dimension. Previous studies also have attempted to examine differences across motivations by gender. Mohsin and Alsawafi (2011) also conclude that gender has a significant impact on some travel motivations. A female preference for shopping is highlighted in Xu et al.'s (2009) findings. Xu et al. (2009) also found differences between male and female youths within each nationality. For example, in the UK, females give higher ratings to 'relaxing' and 'socialising' than males do.

When the relationship between the level of importance given to recreational activities in the destination choices and the push-pull travel motivations; It has been determined that the importance given to recreational activities has a positive and moderate relationship with both push-pull travel motivations. Therefore, based on the findings obtained, as the motivation of push-pull travel motivations increases, the importance given to recreational activities also increases. Likewise, as the level of importance given to recreational activities increases, it can be said that push-pull travel motivations will be positively affected. Tourism activities have received less academic attention previously, while some studies involve activities as components of the picture of destination that attract tourists to the destination (Wang \& Davidson, 2009). From this viewpoint, activities are motivating the destination variables rather than the motivation's behavioral consequences. Likewise, specialist activities are known as factors driving engagement in tourism of special interest at a specific destination (Trauer, 2006). Destination choice based on activity indicates that motivation influences the choice of activities and that visitors will prefer destinations that offer their favorite activities (Moscardo et al., 1996). This model not only clarifies the importance of activities in directing destination choice, but also states a direct relation between travel motivation and activity, for instance, among competition attendees (Kim, Sun \& Mahoney, 2008) or with regard to the positive effect of the risk-taking motive on the purchase of tourism products based on nature (Tangeland et al., 2012). According to Özdemir, Büyüköztürk, and Karaküçük (2016), it can be explained by pull and push travel motives that offer priority to recreational activities when making destination choices. In their research it was recognized that push travel motives were mainly successful in putting emphasis on recreational activities when university students made destination choices.

As critical attributes of destinations, tourism activities and motivations are the main components of leisure trips. 
Understanding of the tourists motivations and activity demands helps to develop the customer profiles, effective positioning and marketing segmentation strategies. Tourism managers can distinguish their services and abilities by understanding the degree to which they meet visitors' needs and expectations. Understanding the value of simultaneous push and pull factors analysis will enable destination managers to stay competitive and increase their market share. There are many opportunities for further research as well. Similar research can be conducted on selected destinations, foreign travelers, different classes of the society, and various occupations and age groups, and cross-cultural comparisons should be planned. There are many similarities and differences among the available motivational concepts, therefore, it necessary to conduct further investigations of travel motives using different tourist groups. Therefore, the destination managers should pay much attention to tourists' travel motives and needs in order to appeal to tourists' internal motives to travel.

\section{References}

Arnett, J. J. (2000). Emerging adulthood: A theory of development from the late teens through the twenties. American Psychologist, 55(5), 469-480. https://doi.org/10.1037/0003-066X.55.5.469

Biederman, P. S. (2008). Travel and tourism: An industry primer. Upper Saddle River, NJ: Pearson/Prentice Hall.

Carr, N. (1999). A study of gender differences: Young tourist behaviour in a UK coastal resort. Tourism Management, 20(2), 223-228. https://doi.org/10.1016/S0261-5177(98)00087-9

Cordes, K. A. (2013). Applications in Recreation \& Leisure: For Today and The Future (4th ed.). Sagamore Publishing. Urbana, IL.

Crompton, J. L. (1979). Motivations for Pleasure Vacation. Annals of Tourism Research, 6(4), 408-424. https://doi.org/10.1016/0160-7383(79)90004-5

Dann, G. (1981). Tourist Motivation: An Appraisal. Annals of Tourism Research, 8(2), 187-219. https://doi.org/10.1016/0160-7383(81)90082-7

Dann, G. M. S. (1979). Anomie, Ego-Enhancement and Tourism. Annals of Tourism Research, 4(4), 184-194. https://doi.org/10.1016/0160-7383(77)90037-8

Field, A. M. (1999). The college student market segment: A comparative study of travel behaviors of international and domestic students at a southeastern university. Journal of Travel Research, 37(4), 375-381. https://doi.org/10.1177/004728759903700407

Gee, C. Y., Mackens, J. C., \& Choy, D. J. (1989). The Travel Industry. New York: Van Nostrand Reinhold.

Iso-Ahola, S. E. (1982). Toward a social psychological theory of tourism motivation: A rejoinder. Annals of Tourism Research, 12, 256-262. https://doi.org/10.1016/0160-7383(82)90049-4

Jackson, E. L., \& Schinkel, D. R. (1981). Recreational activity preferences of resident and tourist campers in the yellow knife region. Canadian Geographer, 25(4), 350-364. https://doi.org/10.1111/j.1541-0064.1981.tb01338.x

Kim, K., \& Jogaratnam, G. (2002). Travel Motivations: A Comparative Study of Asian International and Domestic American College Students. Journal of Travel and Tourism Marketing, 13(4), 61-82. https://doi.org/10.1300/J073v13n04_04

Kim, K., Oh, L. \& Jogaratnam, G. (2007). College Student Travel: A Revised Model of Push Motives. Journal of Vacation Marketing, 13(1), 73-85. https://doi.org/10.1177/1356766706071201

Kim, K., Sun, J., \& Mahoney, E. (2008). Roles of motivation and activity factors in predicting satisfaction: Exploring the Korean cultural festival market. Tourism Analysis, 13(4), 413-425.

Kim, S., \& Lee, C. (2000). Understanding the Cultural Differences in Tourism Motivation between Anglo-American and Japanese Tourists. Journal of Travel and Research Marketing, 9(1/2), 153-170. https://doi.org/10.1300/J073v09n01_09

Kim, S., \& Lee, C. (2002). Push and Pull Relationships. Annals of Tourism Research, 29(1), 257-260. https://doi.org/10.1016/S0160-7383(01)00043-3

Kim, S., Lee, C., \& Klenosky, D. B. (2003). The Influence of Push and Pull Factors at Korean National Parks. Tourism Management, 24, 169-180. https://doi.org/10.1016/S0261-5177(02)00059-6

Klenosky, D. B. (2002). The Pull of Tourism Destinations a Means End Investigation. Journal of Travel Research, 40, 385-395. https://doi.org/10.1177/0047287502040004005

Li, F. (2020). Understanding Chinese tourists' motivations of sharing travel photos in WeChat. Tourism 
Management Perspective, 33, 100584. https://doi.org/10.1016/j.tmp.2019.100584

Mohsin, A., \& Alsawafi, A. M. (2011). Exploring attitudes of omani students towards vacations. Anatolia: An International Journal of Tourism and Hospitality Research, 22(1), 35-46. https://doi.org/10.1080/13032917.2011.556217

Moscardo, G., Morrison, A. M., Pearce, P. L., Lang, C. T., \& O’Leary, J. T. (1996). Understanding Vacation Destination Choice Through Travel Motivation and Activities. Journal of Vacation Marketing, 2(2), 109-122. https://doi.org/10.1177/135676679600200202

Özdemir, A. S. (2013). The Impact of Motivational and Demographic Factors on Destination Choices Based on Recreational Opportunities (Unpublished doctoral dissertation). Gazi University, Institute of Health Sciences, Ankara, Turkey.

Özdemir, A. S., Büyüköztürk, Ş., \& Karaküçük, S. (2016). Rekreasyonel olanaklara dayalı yapılan destinasyon tercihlerinin içsel ve dışsal seyahat motivasyonlarıyla açılanması. Journal of Human Sciences, 13(2), 3002-3021. https://doi.org/10.14687/jhs.v13i2.3920

Pearce, P. L. (1993). Fundamentals of tourist motivation. In D. Pearce, \& R. Butler (Eds.), Tourism Research: Critiques and Challenges (pp. 85-105). Routledge and Kegan Paul: London.

Phau, I., Lee, S., \& Quintal, V. (2013). An investigation of push and pull motivations of visitors to private parks: The case of Araluen Botanic Park. Journal of Vacation Marketing, 19, 269-284. https://doi.org/10.1177/1356766712471232

Richards, G., \& Wilson, J. (2003). Today's youth tourists: Tomorrow's global nomads? New horizons in independent youth and student travel. Amsterdam: International Student Travel Confederation and Arnhem: Association of Tourism and Leisure Education. Retrieved from http://www.atlas-euro.org/pages/pdf/FINAL_Industry_Report.pdf

Rita, P., Brochado, A., \& Dimova, L. (2019). Millennials' travel motivations and desired activities within destinations: A comparative study of the US and the UK. Current Issues in Tourism, 22(16), 2034-2050. https://doi.org/10.1080/13683500.2018.1439902

Ryan, C., \& Glendon, I. (1998). Application of leisure motivation scale to tourism. Annals of Tourism Research, 25(1), 169-184. https://doi.org/10.1016/S0160-7383(97)00066-2

Sakakida, Y., Cole, S. T., \& Card, J. A. (2004). A cross- cultural study of college students' travel preferences: A value-oriented perspective. Journal of Travel \& Tourism Marketing, 16(1), 35-41. https://doi.org/10.1300/J073v16n01_04

Shoham, A., Schrage, C., \& van Eeden, S. (2005). Student travel behavior: A cross-national study. Journal of Travel \& Tourism Marketing, 17(4), 1-10. https://doi.org/10.1300/J073v17n04_01

Tangeland, T., Vennesland, B., \& Nybakk, E. (2012). Second home owners' intention to purchase nature-based tourism activity products: A Norwegian case study. Tourism Management, 36, 364-376. https://doi.org/10.1016/j.tourman.2012.10.006

The World Tourism Organisation. (1995). Concepts, Definition and Classifications for Tourism Statistics (Madrid: World Tourism Organisation). World Travel and Tourism Council, (2006). World Travel and Tourism Clamping to New heights: The 2006 Travel \& Tourism Economic Research.

Thrane, C. (2008). The determinants of students' destination choice for their summer vacation trip. Scandinavian Journal of Hospitality and Tourism, 8(4), 333-348. https://doi.org/10.1080/00220620802588797

Trauer, B. (2006). Conceptualizing special interest tourism frameworks for analysis. Tourism Management, 27(2), 183-200. https://doi.org/10.1016/j.tourman.2004.10.004

Uysal, M., Li, X., \& Sirakaya-Turk, E. (2008). Push-pull dynamics in travel decisions. In O. Haemoon (Ed.), Handbook of Hospitality Marketing Management (pp. 412-439). Butterworth-Heinemann: Oxford. https://doi.org/10.1016/B978-0-08-045080-3.50018-4

Wang, X., \& Walker, G. J. (2010). A comparison of Canadian and Chinese university students' travel motivations. Leisure/Loisir, 34(3), 279-293. https://doi.org/10.1080/14927713.2010.521318

Wang, Y., \& Davidson, M. C. (2009). Chinese leisure tourists: Perceptions and satisfaction with Australia. Tourism Analysis, 14(6), 737-747. https://doi.org/10.3727/108354210X12645141401106

Xiao, H., So K. K. F., \& Wang, A. Y. (2015). The university student travel market: motivations and preferences 
for activities. Tourism Analysis, 20, 399-412. https://doi.org/10.3727/108354215X14400815080488

Xu, F., Morgan, M., \& Song, P. (2009). Students' travel behaviour: A cross-cultural comparison of UK and China. International Journal of Tourism Research, 11, 255-268. https://doi.org/10.1002/jtr.686

Yiamjanya, S., \& Wongleedee, K. (2014). International tourists' travel motivation by push-pull factors and the decision making for selecting Thailand as destination choice. International Journal of Social, Education, Economics and Management Engineering, 8(5), 1348-1353.

\section{Copyrights}

Copyright for this article is retained by the author(s), with first publication rights granted to the journal.

This is an open-access article distributed under the terms and conditions of the Creative Commons Attribution license (http://creativecommons.org/licenses/by/4.0/). 